\title{
Future Development of Endoscopic Accessories for Endoscopic Submucosal Dissection
}

\author{
Jae-Young Jang \\ Division of Gastroenterology, Department of Internal Medicine, College of Medicine, Kyung Hee University, Seoul, Korea
}

Endoscopic submucosal dissection (ESD) has recently been accepted as a standard treatment for patients with early gastric cancer (EGC), without lymph node metastases. Given the rise in the number of ESDs being performed, new endoscopic accessories are being developed and existing accessories modified to facilitate the execution of ESD and reduce complication rates. This paper examines the history underlying the development of these new endoscopic accessories and indicates future directions for the development of these accessories. Clin Endosc 2017;50:242-249

Key Words: Early gastric cancer; Endoscopic submucosal dissection; Endoscopy; Accessory

\section{INTRODUCTION}

Endoscopic resection (ER) techniques are based on the concepts of endoscopic mucosal resection (EMR) and endoscopic submucosal dissection (ESD), which are performed today. The five-year survival rate for patients with early gastric cancer (EGC) who have undergone ER is over $95 \%,{ }^{1,2}$ and ER can lower the risk of postoperative complications, alleviate compromises in quality of life, and reduce morbidity associated with EGC. Consequently, ER is accepted as a standard method for treating EGC that has not metastasized to the lymph nodes. ${ }^{3}$ EMR involves the use of a snare to resect lesions. Tsueneoka et al. performed the first polypectomy that involved the use of a snare in 1967, and this technique was subsequently performed on patients with pedunculated polypoid cancer in 1973. ${ }^{4}$ EMR techniques can include: (1) the inject and cut; (2) the inject, lift, and cut; (3) cap-assisted EMR; and (4) EMR

Received: May 11, 2017 Revised: May 18, 2017

Accepted: May 23, 2017

Correspondence: Jae-Young Jang

Division of Gastroenterology, Department of Internal Medicine, College of Medicine, Kyung Hee University, 23 Kyungheedae-ro, Dongdaemun-gu, Seoul 02447, Korea

Tel: +82-2-958-8260, Fax: +82-2-968-1848, E-mail: jyjang@khu.ac.kr

(c) This is an Open Access article distributed under the terms of the Creative Commons Attribution Non-Commercial License (http://creativecommons.org/ licenses/by-nc/3.0) which permits unrestricted non-commercial use, distribution, and reproduction in any medium, provided the original work is properly cited. with ligation. ${ }^{5}$ However, en bloc resections of lesions larger than $1.5 \mathrm{~cm}$ using EMR are difficult, and as a result, piecemeal resections are performed in some cases. Piecemeal resections cause difficulties in pathological diagnoses, and local recurrence rates as high as $35 \%$ have been reported. ${ }^{5}$ Thus, ESD that enables en bloc resections of large lesions and overcomes shortcomings associated with EMR was developed. ESD was performed for the first time by Hirao et al. in 1988; resecting a lesion involved injecting a hypertonic saline-epinephrine solution around the lesion, making an incision around the lesion with a needle knife, and dissecting the submucosal layer. ${ }^{6}$ This technique is associated with a high risk of perforation, unless it is performed by a skilled endoscopist. To reduce the risk of perforation, Hosokawa et al. developed an insulated-tip (IT) knife, a needle-type knife that has a ceramic ball attached to its tip, and used this knife on 47 patients. ${ }^{7}$ This led to the development of techniques used in ESD today. ESD enables en bloc resections of large lesions that could not be achieved using EMR, and it enables lesions to be resected, irrespective of their location. However, compared with EMR, more time and more assistants are required to perform ESD, and it carries a higher risk of complications, including bleeding and perforation. Nevertheless, some of the shortcomings associated with ESD have been overcome by establishing incision and dissection methods that are appropriate to a lesion's location, increasing endoscopists' experience, improving the techniques 
involved, developing an electrosurgical unit (ESU), and developing a variety of endoscopic accessories, including knives. Endoscopic accessories have undergone radical changes that have substantially simplified ESD and reduced complication rates, and these changes have enabled the rapid management of complications. Consequently, ESD has replaced EMR, and it is now considered a standard ER method. ${ }^{1}$ Endoscopic accessories must be harmless to patients, cheap, strong, and easy to use, and they must increase the complete resection rates, reduce procedural times, and lower complication rates. ER is likely to undergo further development, and it is expected that its popularity will grow. Here, we analyze the history underlying the development of endoscopic accessories for ESD, and we indicate future directions for the development of ESD.

\section{ENDOSCOPIC ACCESSORIES FOR ESD}

\section{Therapeutic endoscopes}

Endoscope development has focused on designing endoscopes that will simplify therapeutic endoscopy procedures, including ESD. A diagnostic endoscope has a working channel that is $2.8 \mathrm{~mm}$ wide, which can complicate suction when a knife or an accessory is present within the channel. Indeed, when bleeding occurs during ESD, the knife must be removed to stop the bleeding, the pool of blood must be suctioned, and water must be injected through a syringe into the area of active bleeding to determine the source of the bleeding. When a hemostatic instrument is subsequently inserted into the working channel, it may be difficult to find the source of the bleeding, due to the amount of blood that has been discharged in the meantime. Repeating this process is time consuming and prolongs the procedure time, which increases the amount of pain experienced by the patient and the rate of complications. Thus, a therapeutic endoscope with a channel wider than $2.8 \mathrm{~mm}$ was developed to overcome these shortcomings; the wider channel enabled suction to be performed without removing accessories. Furthermore, it had a water-jet channel that discharged water when a foot pedal was pressed, which facilitated ascertaining sources of bleeding. However, because of these features, this endoscope had a lower resolution compared with that of the existing diagnostic endoscopes, and it could only produce images that were of standard-definition (SD) quality. Subsequently, single-channel and double-channel endoscopes were developed (Table 1). Endoscopes that feature a double-channel allow 2 instrument to use a choice of channels with regard to optimal angle of approach for dissection, or an open channel for suctioning if only 1 instrument is being used.

\section{Single-channel endoscope}

The distal end of the GIF-Q260J endoscope (Olympus Medical Systems Corp., Tokyo, Japan), which is a single-channel upper gastrointestinal endoscope, measures $9.9 \mathrm{~mm}$ and it has a channel size of $3.2 \mathrm{~mm}$. This endoscope has a water-jet feature, and it provides images that are of SD quality.

\section{Double-channel endoscope}

The double-channel endoscope, GIF-2TQ260M (Olympus Medical Systems Corp., Tokyo, Japan), is available with a multi-bending or double-bending feature (Fig. 1). It has an additional up/down lever. The downward angle can reach $180^{\circ}$, and lesions located in the gastric cardia or in higher parts of the gastric body can be viewed en face. This endoscope has a water-jet feature. The drawbacks associated with this endoscope are its 11.7-mm diameter, which is large, the SD quality images, and its weight $(1.8 \mathrm{~kg})$.

\section{Future directions for endoscope development}

The diagnostic endoscopes that are currently used produce

Table 1. Therapeutic Gastroscope

\begin{tabular}{|c|c|c|c|c|c|c|}
\hline & $\begin{array}{c}\text { GIF-Q260J, } \\
\text { Olympus }\end{array}$ & $\begin{array}{c}\text { GIF-2TQ260M } \\
\text { Olympus }\end{array}$ & $\begin{array}{l}\text { EG-530CT } \\
\text { Fujinon }\end{array}$ & $\begin{array}{l}\text { EG-530D } \\
\text { Fujinon }\end{array}$ & $\begin{array}{c}\text { EG-2990i } \\
\text { Pentax }\end{array}$ & $\begin{array}{c}\text { EG-3890TK } \\
\text { Pentax }\end{array}$ \\
\hline Special light & NBI & NBI & FICE & FICE & i scan & i scan \\
\hline Field of view, ${ }^{\circ}$ & 140 & 140 & 140 & 140 & 140 & 140 \\
\hline Distal end, mm & 9.9 & 11.7 & 10.8 & 11.5 & 9.8 & 12.8 \\
\hline Channel size, $\mathrm{mm}$ & 3.2 & $3.2 / 3.2$ & 3.8 & $3.8 / 2.8$ & 2.8 & $3.8 / 2.8$ \\
\hline Working length, mm & 1,030 & 1,030 & 1,100 & 1,090 & 1,050 & 1,050 \\
\hline Multi bending & No & Yes & No & No & No & No \\
\hline Water jet & Yes & Yes & Yes & Yes & Yes & Yes \\
\hline $\begin{array}{l}\text { Angulation up/down, }{ }^{\circ} \\
\text { right/left, }\end{array}$ & $\begin{array}{c}210 / 90 \\
100 / 100\end{array}$ & $\begin{array}{l}210 / 180 \\
100 / 100\end{array}$ & $\begin{array}{c}210 / 90 \\
100 / 100\end{array}$ & $\begin{array}{c}210 / 90 \\
100 / 100\end{array}$ & $\begin{array}{l}210 / 120 \\
120 / 120\end{array}$ & $\begin{array}{l}180 / 120 \\
120 / 120\end{array}$ \\
\hline
\end{tabular}

NBI, narrow band imaging; FICE, Flexible spectral imaging color enhancement. 


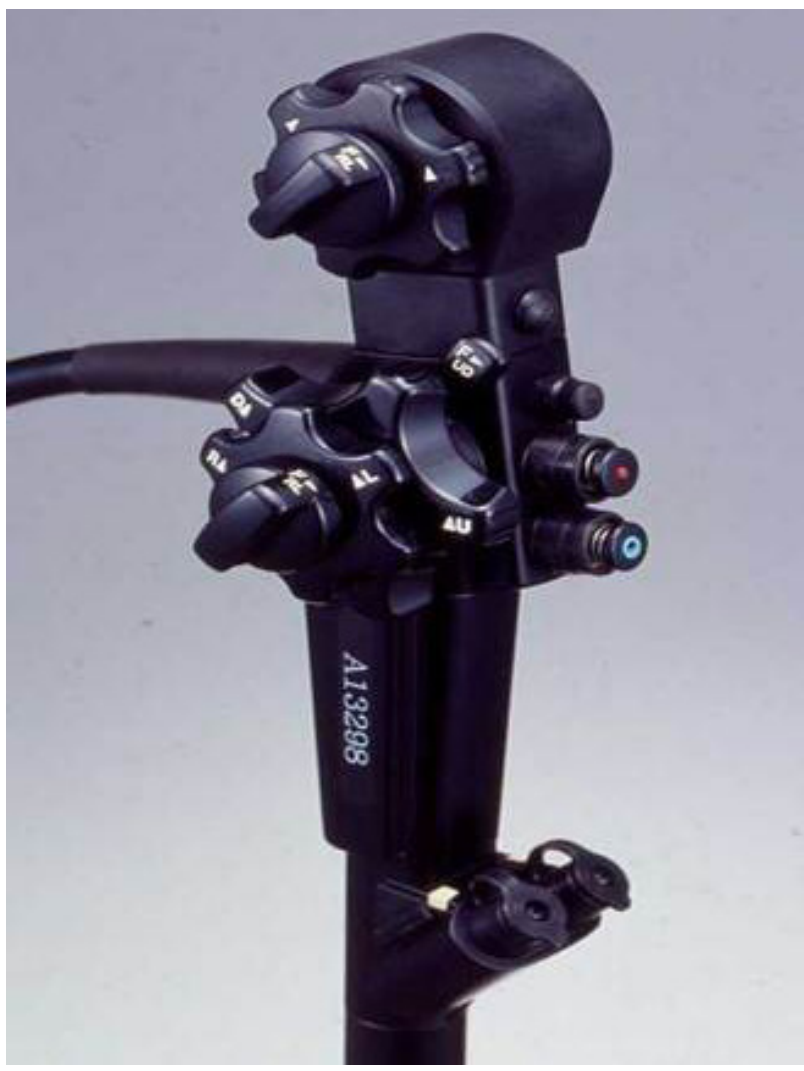

Fig. 1. GIF-2TQ260M (Olympus Medical Systems Corp., Tokyo, Japan). Second bending section overcomes some of the limitations of flexibility inherent with standard endoscopes when approaching anatomically difficult lesions for endoscopic submucosal dissection (ESD).

high definition (HD) and high resolution images, and they enable endoscopists to closely inspect lesions to scrutinize their pit patterns, microvascular patterns, and margins of the lesions even though they are not magnifying endoscopes. Compared with the existing diagnostic endoscopes, it is more difficult to predict the diagnoses and margins of lesions using therapeutic endoscopes, because of their low resolution. The insertion of therapeutic endoscopes is difficult, because the scope's tip has a large diameter that can damage the pharynx or esophagus. Improvements in the diagnosis of EGC and in the treatment outcomes of ESD can be expected when therapeutic endoscopes are developed that retain the therapeutic effects of the existing endoscopes, have a distal end that is less than $1 \mathrm{~cm}$ wide, and provide $\mathrm{HD}$ images.

\section{Injection agents}

"Strip biopsy" is an EMR technique for gastrointestinal mucosal lesions. This improvement in colon polypectomy was introduced in 1984 in Japan. ${ }^{8}$ Because it was difficult to remove flat lesions using conventional polypectomy, lesions were elevated by injecting saline into the submucosal layer, pulled with forceps, and then snared with a trap. The advantages of submucosal injection are that the lesion forms a polypoid shape that can be easily removed, and that the major complication of perforation can be avoided by preventing the snare or knife from directly touching the proper muscle layer.

Submucosal injection solutions include isotonic saline, sodium hyaluronate $(\mathrm{SH})$, hypertonic saline (3.8\%), hypertonic glucose $(20 \%, 50 \%)$, and a glycerin mixture $(10 \%$ glycerin $+5 \%$ fructose $+0.9 \% \mathrm{NaCl}$ ). One should consider the elevation capacity of the tissue, the total duration of elevation, the potential for tissue injury, the hemostatic capacity of the tissue, the cost, and other factors when selecting the submucosal injection solution. Normal saline as an isotonic solution is inexpensive and harmless to the tissue. However, because it is not good at maintaining elevation and lacks hemostatic capacity, it is often mixed with epinephrine and indigo carmine when it is used. The hypertonic solutions, hypertonic saline and hypertonic glucose, are seldom used because they can injure the tissue, although they are good at maintaining elevation. The glycerin mixture is advantageous because it seldom injures the tissue, as it is a mildly hypertonic solution with osmotic pressure only two-fold above physiological osmotic pressure. It exhibits a relatively good capacity to maintain elevation and is inexpensive. It is used in combination with epinephrine, but depending on individual preferences, it can be mixed with indigo carmine. Compared with other submucosal injection solutions, $\mathrm{SH}$ solution is considered ideal due to its high viscoelasticity, but it has the disadvantage of being costly. Depending on molecular weight, two types of $\mathrm{SH}$ solution are available commercially ( $800 \mathrm{kDa}$ and $1900 \mathrm{kDa})$. SH should be diluted to a concentration that maintains both its viscoelasticity and its capacity to maintain mucosal elevation. A $0.4 \%$ SH solution is widely used due its capacity to maintain elevation, although its high cost is a limitation. Recent research indicated a paradigm shift toward the development of mucosal resection injection solutions that rely on gel formation to provide extended submucosal lift durations. ${ }^{9-14}$

\section{Sodium alginate}

Sodium alginate (SA) derived from brown algae has been clinically used in anti-peptic ulcer agents. Kusano et al. reported the utility of SA solution as a novel endoscopic submucosal injection material in ESD. ${ }^{9}$ Compared with $0.4 \%$ SH solution, $0.6 \%$ SA solution exhibited no significant difference in catheter injectability but significant superiority in mucosa-elevating capacity. In the clinical study, no adverse events were observed in any patient. The results warrant further investigation in a large patient population to evaluate the usefulness of this material as a submucosal injection solution for gastric ESD. 


\section{Photocrosslinked chitosan hydrogel}

Photocrosslinkable chitosan and thermoresponsive poloxamers have been recently reported for EMR with great enthusiasm. ${ }^{10}$ Chitosan containing $2 \%$ lactobionic acid exhibited high aqueous solubility, even at neutral $\mathrm{pH}$. Furthermore, application of ultraviolet light irradiation at a lamp distance of 2 $\mathrm{cm}$ with a guide fiber unit and a 250 -W lamp to $2.5 \% \mathrm{p}$-azidebenzoic acid produced an insoluble hydrogel within $30 \mathrm{sec}-$ onds and firmly adhered two pieces of ham to each other. The liquid-to-gel transformation using photoinitiated free radical polymerization requires the use of ultraviolet light, which may be difficult in hard-to-reach areas, and thermoresponsive polymers have been shown to clog inside long delivery tools at normal body temperature.

\section{Thermally sensitive injection agents}

Recombinant peptide polymers, such as elastin-like polypeptides (ELPs), provide an attractive route for the design of such materials because they are injectable, biodegradable, biocompatible, and nonimmunogenic. ${ }^{11}$ ELPs are attractive as injectable biomaterials undergoing a soluble to insoluble phase transition when heated above a tunable transition temperature. ELP exhibited an equivalent effectiveness of mucosal elevation, retention of elevation, and minimal bleeding with $\mathrm{SH}$. Another thermo-sensitive colloid was also designed; poly(lactic acid-co-glycolic acid)-poly(ethylene glycol)-poly(lactic acid-co-glycolic acid) (PLGA-PEG-PLGA) thermogel is soluble at room temperature and relatively easy for endoscopic injection. The polymer/water system is a low-viscosity fluid at room temperature and thus is easily injected, and turns into a non-flowing gel at body temperature after injection. ${ }^{12}$ High mucosal elevation with a clear margin was maintained for a long duration. The PLGA-PEG-PLGA thermogel provides an excellent submucosal injection system, and has great potential to significantly improve the ESD technique.

\section{Mesna}

Recently, injectables with autodissection properties have been evaluated. Mesna (sodium 2-sulfanylethanesulfonate) is a thiol compound that dissolves disulfide bonds in connective tissue between anatomic planes and has been shown to be useful for chemical dissection in surgical fields. ${ }^{13}$ Mesna was evaluated in a double-blind, randomized, placebo-controlled trial of 101 patients undergoing gastric ESD. ${ }^{14}$ Submucosal dissection time was 18.6 minutes in the mesna group and 24.6 minutes in the placebo group, a difference that did not achieve statistical significance. However, multivariate regression analysis found use of mesna to correlate strongly with submucosal dissection time. The role of mesna in facilitating ESD requires further evaluation at this time.

\section{Future directions for injection agent development}

The ideal injection solution should be cost-effective, widely available, easily injectable, biocompatible, biodegradable, able to provide prolonged submucosal lift durations, and able to aid in mucosal healing after the resection process to have clinical relevance. So far, no injection fluid is widely accepted as the optimal agent for ESD. Further studies will be required to improve its biosafety and effectiveness for clinical application.

\section{Knives}

The development of knives underpinned the introduction of ESD. Consequently, knives are the most fundamental ESD accessory.

\section{Needle-type knives}

A needle-type knife (KD-1L-1; Olympus Medical Systems Corp., Tokyo, Japan), which was developed in 1988, can mark, incise, and dissect lesions; however, its sharp tip and the electric current that flows into its tip increase the risk of perforation. ${ }^{6}$ Accordingly, a flex knife (KD-630L; Olympus Medical Systems Corp., Tokyo, Japan) was developed to overcome the shortcomings associated with the needle-type knife. The flex knife has a round tip that consists of twisted wire that is like a snare, and its soft and flexible sheath prevents perforations. However, carbonized particles tend to stick to the tip and hinder flow of the electric current into the tip, which complicates procedures. This limitation was overcome by the development of a dual knife (KD-650L/KD-650Q; Olympus Medical Systems Corp., Tokyo, Japan), which has a small $(0.3 \mathrm{~mm})$ balllike process on the top that prevents it from slipping. Only the ball-like process protrudes when the knife is retracted, which facilitates lesion marking. To prevent perforations, the knife's length is fixed at $2 \mathrm{~mm}$ in the stomach and at $1.5 \mathrm{~mm}$ in the esophagus and colon.

\section{Insulated-tip knife}

The IT knife (KD-610L; Olympus Medical Systems Corp., Tokyo, Japan) that was developed in 1998 is covered by a ceramic ball; the electric current does not flow into its tip, which prevents perforations during incision and dissection. ${ }^{7}$ However, it is impossible to mark lesions using an IT knife, and an incision can only be made when a hole is precut using a needle-type knife or a snare's tip. Although the risk of perforation is reduced, the knife is difficult to handle. Making an incision and performing a dissection with the IT knife are straightforward only when the angle between the mucous membrane and the knife is $30^{\circ}-60^{\circ}$. While an IT knife is more difficult for trainee endoscopists to use than a needle-type knife, incisions can be made and dissections can be performed rapidly once a user has become more accustomed to handling the 
knife. An IT knife was developed that had a triangle-shaped electrode at the bottom of the ceramic ball to improve incision and dissection (IT knife 2, KD-610L; Olympus Medical Systems Corp., Tokyo, Japan), and this was followed by the development of the IT knife nano (KD-612U/L; Olympus Medical Systems Corp., Tokyo, Japan), which can be used on lesions in the esophagus and colon.

\section{Knives that enable submucosal injections}

To perform a submucosal injection while making an incision or performing a dissection, the endoscopist must removing the knife and insert an injector, which is a cumbersome process. To alleviate the inconvenience associated with this process, a knife was developed that is equipped with a water-jet channel that can inject water through a syringe attached to the knife or a water pump. The flush knife (DK2618JB/DK2618JN; Fujifilm Corporation, Tokyo, Japan) is a needle-type knife that is available in five different lengths, namely, 1, 1.5, $2,2.5$, and $3 \mathrm{~mm}$. A knife of an appropriate length is used depending on the characteristics of the lesion. A conduction pump is used for the injection. The dual knife J (KD-655L/ Q/U; Olympus Medical Systems Corp., Tokyo, Japan) has a water-jet hole, and water is injected through a syringe that is located next to the handle. A relatively large amount of pressure must be applied to inject water into the submucosal layer. A hybrid knife (20150-060, 061, 062; Erbe Elektromedizin $\mathrm{GmbH}$, Tübingen, Germany) is available in T, I, and O types, which vary according to the shape of the knife's tip, and there is a central capillary within the knife that executes the submucosal injection through a water-jet system when a foot pedal is pressed. Given the force required, the submucosal injection can be performed when the knife is placed against the mucous membrane and the foot pedal is pressed; however, a jet lavage unit (Erbejet 2 system; Erbe Elektromedizin $\mathrm{GmbH}$, Tübingen, Germany) has to be additionally purchased.

\section{Other knives used in endoscopic submucosal dissection}

Other knives used in ESD that have different shaped tips include the hook knife (KD-620LR/KD-620QR; Olympus Medical Systems Corp., Tokyo, Japan), the triangle-tip (TT) knife (KD-640L; Olympus Medical Systems Corp., Tokyo, Japan), and the scissor type SB knife (MD-47706/MD-47704 and MD-47703; Akita Sumitomo Bakelite Co., Ltd, Akita, Japan).

\section{Future directions for knife development}

Although different shaped knives have been developed, most are now over 10 years old. The recent trend in knife development has been to modify existing knives to produce knives that overcome their predecessors' shortcomings and simplify the ESD procedures, rather than to develop knives with new shapes. An endoscopist undergoing training in ESD uses a single type of knife until they become proficient, and it is difficult for them to subsequently switch to differently shaped knives. Needle-type knives will be fabricated in such a way that only one type of knife will be required to perform all of the steps that comprise ESD. An IT knife is more difficult to handle than the needle-type knives, and this is a shortcoming that needs to be addressed. Indeed, an IT knife must be developed that can mark and perform submucosal injections in ways that are similar to needle-type knives. Furthermore, we hope that knives will be made out of materials that are harmless to patients and that do not require cleaning to remove carbonized particles from their tips.

\section{Transparent caps}

The attachment of a transparent cap to the tip of an endoscope enables an "en face" approach to be undertaken for oblique lesions or lesions in blind areas. By fixing the endoscope to the mucous membrane of a lesion, the tip of the endoscope can be stabilized and a distance of 3-4 $\mathrm{mm}$ can be maintained, even when the tip of the endoscope comes into contact with the lesion; hence, the endoscopist's vision is safeguarded. The findings from a study of 19 patients whose bleeding peptic ulcers underwent hemostasis demonstrated the effectiveness of transparent caps when they are fitted to endoscopes. ${ }^{15}$ Transparent caps attached to endoscopes have been useful in ESDs. Performing a dissection without checking the dissection plane is associated with a high risk of bleeding and perforation; therefore, it is important to closely monitor the dissection plane during the dissection. Attaching a transparent cap to the tip of an endoscope enables hemostatic procedures to be performed in advance on vessels that are at a high risk of bleeding, the thickness of the submucosal layer to be resected to be controlled, and dissection of the submucosal layer parallel to the muscle layers, thereby preventing perforations. Hence, attaching a transparent cap to the tip of an endoscope is considered an essential part of ESD.

\section{Transparent distal attachment}

A transparent distal attachment (D-201; Olympus Medical Systems Corp., Tokyo, Japan) can provide a large, general field of view. Furthermore, the transparent distal attachment is made of soft and smooth materials, which enable it to expose the submucosal layer by lifting the mucous membrane without damaging the muscle layers, and to apply light pressure to the submucosal layer. The 4-mm-long cap protrudes from the tip of the endoscope, and the diameter of the cap varies from $10.7 \mathrm{~mm}$ to $14.3 \mathrm{~mm}$. Recently, drilling a hole on the external side of the cap solved the problem of water or blood collecting 
at the tip of the endoscope. The hole is drilled on the opposite side of the suction channel to enable water or blood that is not easily removed through aspiration to drain naturally.

\section{Small-caliber-tip transparent hood}

The cone-shaped small-caliber-tip transparent (ST) hood (DH-15GR and DH-15CR; Fujifilm Corporation, Tokyo, Japan) is useful for making and opening an incision in the mucous membrane of the stomach or intestine and resecting the lesion while facing the submucosal layer without damaging the muscle layers. The tip of the hood is $7 \mathrm{~mm}$ long, and the hood tapers towards the tip where the diameter is $1 \mathrm{~mm}$, which facilitates elevation of mucous membranes. When it is difficult to see the submucosal layer in the intestine or in the body of the stomach, or fibrosis is present that demands a sophisticated dissection, the ST hood can be used to easily penetrate the submucosal layer and safely scrutinize the submucosa and blood vessels. However, the ST hood restricts the field of view, and it is difficult to monitor the incision and its surrounding tissue.

\section{Future directions for transparent cap development}

Transparent caps are not completely transparent and they limit the field of view when they are attached to an endoscope. Identifying the sources of bleeding on the dissection plane will become more straightforward when the transparency of these caps increases, and a thinner tip will facilitate the penetration of the endoscope into the dissection plane in areas of severe fibrosis. Furthermore, the transparent caps are not sufficiently soft and when contact is made with the mucous membrane during fixation to a lesion, the caps can cause injuries and bleeding. We anticipate the development of transparent caps with improved transparency and flexibility, and the development of different shaped caps for lesions that are in different locations and have varying degrees of fibrosis.

\section{Hemostatic forceps}

Bleeding is a complication that cannot be avoided during ESD, and it can occur during all endoscopic procedures. Although bleeding stops naturally in most cases, bleeding from large veins or arteries can interfere with the endoscopist's vision during an ESD, causes pain to the patient, and prolongs the procedural time if it is not stopped immediately. Bleeding that involves blood oozing from small veins can be stopped with a knife. When pulsating bleeding or bleeding from a large blood vessel occurs on the incision or dissection plane, it is difficult to stop the bleeding with a knife, and other hemostatic instruments must be used. It is difficult to stop pulsating bleeding using argon plasma coagulation, and heater probe coagulation, which stops the bleeding by applying pressure to the blood vessel, can damage the muscle layers and increase the risk of perforation. Consequently, a hemostatic procedure in which a hot biopsy forceps is used to hold and pull a blood vessel to reduce its contact with the muscle layers was developed. The hot biopsy forceps has a concave wing that enables it to hold a polyp. However, the area of contact between the hot biopsy forceps and the blood vessel was insufficient, and hemostatic forceps (Coagrasper FD-410LR and FD-412LR; Olympus Medical Systems Corp., Tokyo, Japan and HDB2418; Pentax Medical, Tokyo, Japan) with a wing that is flat on its inner side was subsequently developed. When the forceps are retracted, the tip becomes a circular rod that applies pressure to the site and stops the bleeding.

\section{Traction devices}

A dissection must begin from the direction in which the lesion can be easily exposed from the dissection plane through gravity. A lesion must be dissected from the proximal region when located on the greater curvature side of the gastric antrum, and from the distal region when located on the lesser curvature side of the gastric angle and body. However, it is difficult to expose the submucosal layer of large lesions, lesions that are accompanied by ulcers, and lesions that are located on the greater curvature of the gastric body and pylorus, and the resection becomes technically challenging. This results in prolonged procedural times and increased risks of complications. Accordingly, the traction method in which the submucosal layer is exposed to provide a clear view of the cutting line and the submucosal vessels was proposed. Methods that used the existing endoscopic accessories have been described in a case series. ${ }^{16}$ The clip-with-line method, in which a thread is attached to a clip and inserted and the edge of the lesion is held by the clip and subjected to traction by the thread, the external forceps method, in which an external grasping forceps is inserted into the working channel and is used to hold another grasping forceps or a snare to pull the edge of the lesion, and

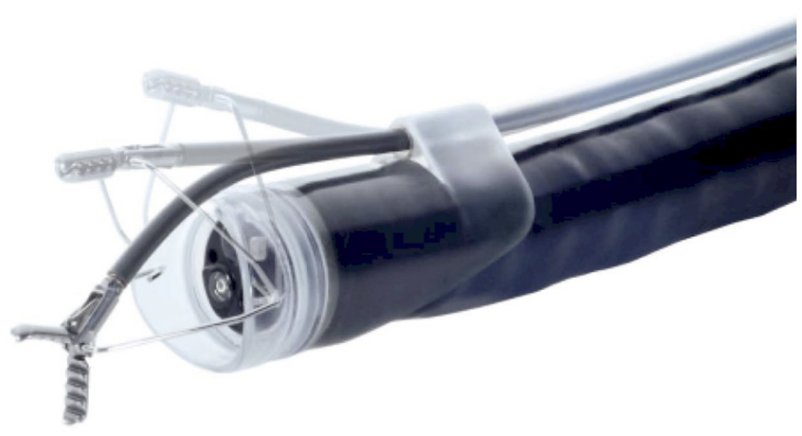

Fig. 2. EndoLifter (LA-201; Olympus Medical Systems Corp., Tokyo, Japan). Traction applied with the external forceps can elevate the lesion and make the submucosal layer wider and more visible. 
the clip-and-snare method have been described. The double scope method, which involves inserting two endoscopes, namely, a small caliber scope that is used to pull the lesion and a scope that is used for dissection, has also been proposed. ${ }^{17}$ The EndoLifter (LA-201; Olympus Medical Systems Corp., Tokyo, Japan) is a grasping forceps with a transparent cap that is much easier to insert compared with other accessories (Fig. 2). The findings from one study showed that while an EndoLifter did not affect the procedure time, the submucosal layer could be better visualized in the group in which an EndoLifter was used. $^{18}$

\section{Future directions for the development of the traction method}

Experienced endoscopists can perform ESDs quickly and safely without using the traction method. The traction method should be used by relatively inexperienced endoscopists and trained endoscopists to shorten procedural times for lesions that have severe fibrosis and to prevent complications. Most of the research into the traction method has focused on testing the efficacy of the method, and no studies have been undertaken that directly compare it with other methods. The insertion of traction accessories while using a grasping forceps or a snare that is attached to an endoscope is difficult and it can cause injuries. The development of traction accessories that are easier to insert, channels through which traction accessories can be inserted, or traction accessories that are much easier to handle is anticipated.

\section{Other devices and instruments used in ESD}

\section{Thulium laser system}

The thulium laser system emits a laser with a $2-\mu \mathrm{m}$ wavelength that enables ESDs. The theoretical penetration depth of $0.25 \mathrm{~mm}$ reduces the risk of perforation, and all of the steps that comprise ESD can be performed using the thulium laser system. The findings from a study of 10 patients treated with gastric ESD showed that, compared with ESD undertaken using a knife, ESD involving the use of the thulium laser system was associated with longer incision times and more difficult resections for lesions with severe fibrosis. ${ }^{19}$ Although the thulium laser system is effective, it has a number of shortcomings that are described here. First, the incisions made with the thulium laser system are not as smooth as those that are made with a knife. Second, the mucous membrane surrounding the incision can be severely burned. Third, a laser system must be purchased, which is quite costly. If the wavelength of the laser can be controlled for incisions, dissections, and coagulation using different settings like those on an ESU, ESDs will be performed much more easily. We anticipate the development of other laser systems that will perform ESDs much more easily and safely than the thulium laser system.

\section{The master and slave transluminal endoscopic robot}

The Master and Slave Transluminal Endoscopic Robot (MASTER) is an endoscope that is equipped with robotic arms, developed in 2012; dissections using the MASTER were performed on five patients with EGC. ${ }^{20}$ The MASTER is comprised of a human-master robotic interface, a telesurgical workstation, and slave manipulator. The most critical component of the robotic system is the slave manipulator, since it is the portion of the robotic system that performs the surgery on the human body. The slave manipulator was mounted on a forward viewing therapeutic endoscope with 2 operating channels (GIF-2T240; Olympus, Co, Ltd, Tokyo, Japan). The slave manipulators control the end effectors, which include a monopolar diathermy "L" shaped hook and a grasper (Fig. 3). An endoscopist holds the endoscope, while the surgeon controls the master interface and operates the slave manipulator to perform the treatment. Using a different endoscope, the lesion is marked, the submucosal injection is administered, and the incision is made while the patient is under general anesthesia; then, the double-channel endoscope with robotic arms is inserted to perform the dissection. Since it is difficult for robots to perform these sophisticated tasks, the MASTER

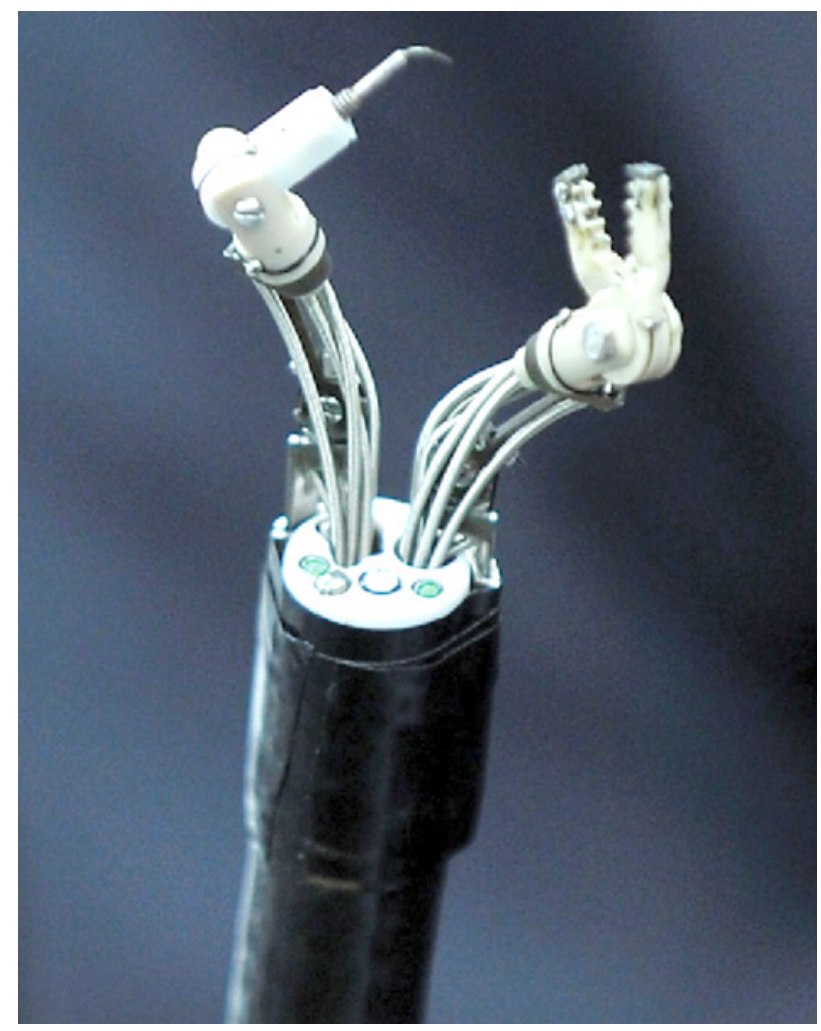

Fig. 3. The slave manipulator of the master and slave transluminal endoscopic robot (MASTER) system. 
remains at an experimental stage, and its use is inconvenient currently. Indeed, when a submucosal injection is required, a different endoscope must be used to perform these tasks, and the MASTER has to be reinserted. However, we are at the prelude to an era of robotic therapeutic endoscopy, and the MASTER provides insights into the future and has infinite potential for development. While we can control robotic arms that are attached to an endoscope, the development of endoscopes and accessories that can be remotely operated in a sophisticated manner will mark a new milestone for ESD and for the entire field of therapeutic endoscopy.

\section{AREAS OF FUTURE RESEARCH}

Most recent research has scrutinized the efficacy and feasibility of recently developed accessories. Studies that have directly compared knives, transparent caps, and traction methods are rare. A large, prospective, multicenter study that generates data that will guide the future direction of the development of accessories must be conducted. Moreover, research into the development of an ESD training system and equipment for beginners and the cost-effectiveness of the improved accessories must be conducted.

\section{CONCLUSIONS}

Cancer stages are currently determined based on the resected specimen. Since future treatment directions and follow-up intervals are determined based on the staging results, lesions will continue to be carefully resected for staging rather than being ablated. As endoscopists become more experienced in the use of the variety of accessories available and they become familiar with the different ways to approach lesions at different locations, complications rates will fall, procedural times will shorten, and the en bloc resection rates will increase. Endoscopic accessories will continue to be developed and will improve, and ESD will become a relatively easy procedure in the future. These endeavors will lay the foundation for the development of robotic therapeutic endoscopy beyond ESD.

\section{Conflicts of Interest}

The author has no financial conflicts of interest.

\section{REFERENCES}

1. Isomoto H, Shikuwa S, Yamaguchi N, et al. Endoscopic submucosal dissection for early gastric cancer: a large-scale feasibility study. Gut 2009;58:331-336.

2. Ahn JY, Jung HY, Choi KD, et al. Endoscopic and oncologic outcomes after endoscopic resection for early gastric cancer: 1370 cases of absolute and extended indications. Gastrointest Endosc 2011;74:485-493.

3. Gotoda T. Endoscopic resection of early gastric cancer. Gastric Cancer 2007;10:1-11.

4. Oguro Y. Endoscopic treatment of early gastric cancer. Dig Endosc 1991;3:3-15.

5. Soetikno RM, Gotoda T, Nakanishi Y, Soehendra N. Endoscopic mucosal resection. Gastrointest Endosc 2003;57:567-579.

6. Hirao M, Masuda K, Asanuma T, et al. Endoscopic resection of early gastric cancer and other tumors with local injection of hypertonic saline-epinephrine. Gastrointest Endosc 1988;34:264-269.

7. Ohkuwa M, Hosokawa K, Boku N, Ohtu A, Tajiri H, Yoshida S. New endoscopic treatment for intramucosal gastric tumors using an insulated-tip diathermic knife. Endoscopy 2001;33:221-226.

8. Tada M, Shimada M, Murakami F, et al. Development of the strip-off biopsy. Gastroenterological Endoscopy 1984;26:833-839.

9. Kusano T, Etoh T, Akagi T, et al. Evaluation of $0.6 \%$ sodium alginate as a submucosal injection material in endoscopic submucosal dissection for early gastric cancer. Dig Endosc 2014;26:638-645.

10. Ishihara M, Kumano I, Hattori H, Nakamura S. Application of hydrogels as submucosal fluid cushions for endoscopic mucosal resection and submucosal dissection. J Artif Organs 2015;18:191-198.

11. Liu W, Zhao M, Liu W, Zheng Z, Zhang X. A feasibility study of a thermally sensitive elastin-like polypeptide for submucosal injection application in endoscopic resection in 3 animal models. Gastrointest Endosc 2015;82:944-952.

12. Yu L, Xu W, Shen W, et al. Poly(lactic acid-co-glycolic acid)-poly(ethylene glycol)-poly(lactic acid-co-glycolic acid) thermogel as a novel submucosal cushion for endoscopic submucosal dissection. Acta Biomater 2014;10:1251-1258.

13. Benassi L, Lopopolo G, Pazzoni F, et al. Chemically assisted dissection of tissues: an interesting support in abdominal myomectomy. J Am Coll Surg 2000;191:65-69.

14. Sumiyama K, Toyoizumi H, Ohya TR, et al. A double-blind, block-randomized, placebo-controlled trial to identify the chemical assistance effect of mesna submucosal injection for gastric endoscopic submucosal dissection. Gastrointest Endosc 2014;79:756-764.

15. Yap CK, Ng HS. Cap-fitted gastroscopy improves visualization and targeting of lesions. Gastrointest Endosc 2001;53:93-95.

16. Kume K. Endoscopic therapy for early gastric cancer: standard techniques and recent advances in ESD. World J Gastroenterol 2014;20:64256432.

17. Tsuji K, Yoshida N, Nakanishi H, Takemura K, Yamada S, Doyama H. Recent traction methods for endoscopic submucosal dissection. World J Gastroenterol 2016;22:5917-5926.

18. Teoh AY, Chiu PW, Hon SF, Mak TW, Ng EK, Lau JY. Ex vivo comparative study using the Endolifter ${ }^{\circledR}$ as a traction device for enhancing submucosal visualization during endoscopic submucosal dissection. Surg Endosc 2013;27:1422-1427.

19. Cho JH, Cho JY, Kim MY, et al. Endoscopic submucosal dissection using a thulium laser: preliminary results of a new method for treatment of gastric epithelial neoplasia. Endoscopy 2013;45:725-728.

20. Phee SJ, Reddy N, Chiu PW, et al. Robot-assisted endoscopic submucosal dissection is effective in treating patients with early-stage gastric neoplasia. Clin Gastroenterol Hepatol 2012;10:1117-1121. 